\title{
Event boundaries and anaphoric reference
}

\author{
Alexis N. Thompson ${ }^{1} \cdot$ Gabriel A. Radvansky ${ }^{1}$
}

Published online: 9 October 2015

(C) Psychonomic Society, Inc. 2015

\begin{abstract}
The current study explored the finding that parsing a narrative into separate events impairs anaphor resolution. According to the Event Horizon Model, when a narrative event boundary is encountered, a new event model is created. Information associated with the prior event model is removed from working memory. So long as the event model containing the anaphor referent is currently being processed, this information should still be available when there is no narrative event boundary, even if reading has been disrupted by a working-memory-clearing distractor task. In those cases, readers may reactivate their prior event model, and anaphor resolution would not be affected. Alternatively, comprehension may not be as event oriented as this account suggests. Instead, any disruption of the contents of working memory during comprehension, event related or not, may be sufficient to disrupt anaphor resolution. In this case, reading comprehension would be more strongly guided by other, more basic language processing mechanisms and the event structure of the described events would play a more minor role. In the current experiments, participants were given stories to read in which we included, between the anaphor and its referent, either the presence of a narrative event boundary (Experiment 1) or a narrative event boundary along with a workingmemory-clearing distractor task (Experiment 2). The results showed that anaphor resolution was affected by narrative event boundaries but not by a working-memory-clearing
\end{abstract}

Gabriel A. Radvansky

gradvans@nd.edu

1 Department of Psychology, University of Notre Dame, Notre Dame, IN 46556, USA distractor task. This is interpreted as being consistent with the Event Horizon Model of event cognition.

Keywords High order cognition · Language comprehension . Mental models · Event Horizon Model · Situation models . Event models $\cdot$ Anaphor processing

During language comprehension, a continuous stream of information can be parsed through a process of event segmentation that can have a later influence on memory. For example, there may be a memory decline for information carried across boundaries from one event to another (Lawrence \& Peterson, 2014; Radvansky \& Copeland, 2006, 2010; Radvansky, Krawietz, \& Tamplin, 2011; Radvansky, Pettijohn, \& Kim, 2015; Radvansky, Tamplin, \& Krawietz, 2010). Conversely, in other cases, there is an improvement of memory for information that occurs more proximal to an event boundary (e.g., Swallow, Zacks, \& Abrams, 2009) and is separated by event boundaries (e.g., Zacks \& Tversky, 2001). The focus here is on memory for information that is outside the current event during text comprehension (e.g., Glenberg, Meyer, \& Lindem, 1987; Rinck \& Bower, 1995; Zwaan, 1996). Specifically, we assessed the resolution of anaphors as a function of the presence or absence of event boundaries. More specifically, we aimed to assess whether a decrease in the availability of information outside of the current event is due to the segmentation of elements into different events or a more general removal of information from working memory.

The segmentation of the flow of information into event models is well documented. The event indexing model (Zwaan, Magliano, \& Graesser, 1995b; Zwaan \& Radvansky, 1998) identifies at least five dimensions that readers track during comprehension (i.e., space, time, entities, causality, and intentionality). The segmentation of event information is 
supported by research showing that reading times tend to increase at these event boundaries (e.g., Zwaan, Magliano, \& Graesser, 1995b; Zwaan, Radvansky, Hilliard, \& Curiel, 1998), which has been interpreted as reflecting an increase in cognitive effort as readers update their event models. Even when reading time data fails to reflect such an updating, other measures, such as probe response times, show that such segmentation and updating is occurring (Radvansky \& Copeland, 2010).

Aside from reading times, event segmentation has been shown using overt segmentation (e.g., Magliano, Kopp, McNerney, Radvansky, \& Zacks, 2012; Zacks, Speer, \& Reynolds, 2009), often using a variant of the Newtson task (e.g., Newtson, 1973.), sorting tasks (Zwaan, Langston, \& Graesser, 1995a), probe identification tasks (e.g., Radvansky, Copeland, Berish, \& Dijkstra, 2003; Zwaan, 1996), and priming studies (Ezzyat \& Davachi, 2011). Moreover, this sort of event segmentation applies not only to reading comprehension but also to the comprehension and processing of film (e.g., Magliano \& Zacks, 2011; Zacks et al., 2009) and interactive virtual environments (e.g., Copeland, Magliano, \& Radvansky, 2006; Tamplin, Krawietz, Radvansky, \& Copeland, 2013).

It has been demonstrated repeatedly that event boundaries can result in a processing cost for accessing information that was mentioned prior to encoding an event boundary. In many cases, a probe recognition tasks is used in which reading is interrupted with a memory probe to assess the accessibility of prior text information. Typically, that information is less available if there was a narrative event boundary between the memory probe and the probed-for information (Bower \& Morrow, 1990; Glenberg et al., 1987; Levine \& Klin, 2001; Morrow, Bower, \& Greenspan, 1989; Morrow, Greenspan, \& Bower, 1987; Radvansky \& Copeland, 2010; Speer \& Zacks, 2005; Zwaan, 1996).

While such studies are informative, a better way to assess comprehension is to not disrupt normal reading so that we can be more confident that the observed effects are not due to postor extra-comprehension processes. To do this, we used reading times to assess the effectiveness of processing anaphors as a function of the influence of event boundaries. This general topic has been explored by other research (Anderson, Garrod, \& Sanford, 1983; Claus \& Kelter, 2006; Glenberg et al., 1987; Kelter, Kaup, \& Claus, 2004; Radvansky \& Copeland, 2001; Rinck \& Bower, 1995). This work has found that when an event boundary is encountered in a text, people are slower and less accurate to resolve anaphors that refer to referents that were part of the prior event but not the current event.

For our study, we explored whether the effect of a narrative event boundary on anaphor resolution is due to (a) the separation of information into different event models or (b) a general process, not strongly tied to event structure, involving the removal of information from working memory. The segmentation of information into separate event models was done via the presence of event boundaries in a text. In comparison, the removal of information from working memory is done by providing people with a 30 -second distractor task of twodigit multiplication problems. Each of these possibilities is considered in turn (see Fig. 1).

One possibility in a decline in information availability following a narrative event boundary is that the boundary segregates the information into different mental structures, or chunks, namely, event models. According to the Event Horizon Model (Radvansky, 2012; Radvansky \& Zacks, 2011, 2014), when an event boundary is encountered, it causes information to be put into separate event models. More importantly, information that is in the current event model is held in working memory. In contrast, information that is part of the previous event model is removed from working memory, rendering it less accessible. If comprehension were interrupted with a distractor task that cleared out working memory between reading the referent and the anaphor, then when reading resumed, the event model representing the text prior to the disruption would be returned to working memory, and the accessibility of the anaphor referent should be similar to when comprehension is not disrupted. In this case, anaphor resolution would be worse when there is a narrative event boundary but not if there is only a distractor task.

Another possibility is that it is not the narrative event boundary per se causing a disruption in anaphor resolution but the removal of information from working memory. The boundary sentence may not result in the creation of a new event model but may instead signal the removal of some information from working memory (Kintsch, 1988). From this view, anaphor resolution is harder because those concepts are not maintained in working memory. Such a cessation of information maintenance in working memory may reflect more general cognitive processes not strongly tied to event structure per se. For such a view, narrative event boundaries are cues to stop maintaining information in working memory. If this is the case, then any other actions taken during reading that also have the effect of clearing out working memory should have a similar effect. Thus, if reading were disrupted with a distractor task that cleared out working memory, then the anaphor resolution difficulty would be present both with a narrative event boundary and with a distractor task, although they may not necessarily be of the same magnitude. In both of these cases, the reader would need to access any referent information that might be available in long-term memory.

There has been prior work assessing reading interruptions by a distractor task during narrative comprehension, although primarily with expository rather than narrative texts. This work has focused on the ability to answer recognition questions and has found that the disruption did not impair performance (Fischer \& Glanzer, 1986; Glanzer, Dorfman, \& Kaplan, 1981; Glanzer, Fischer, \& Dorfman, 1984; Oulasvirta \& Saariluoma, 2006). However, in most of those studies, the postreading memory test involved verifying facts from 


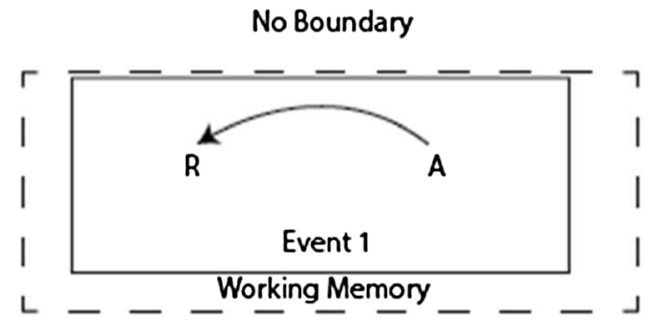

Event Horizon Model

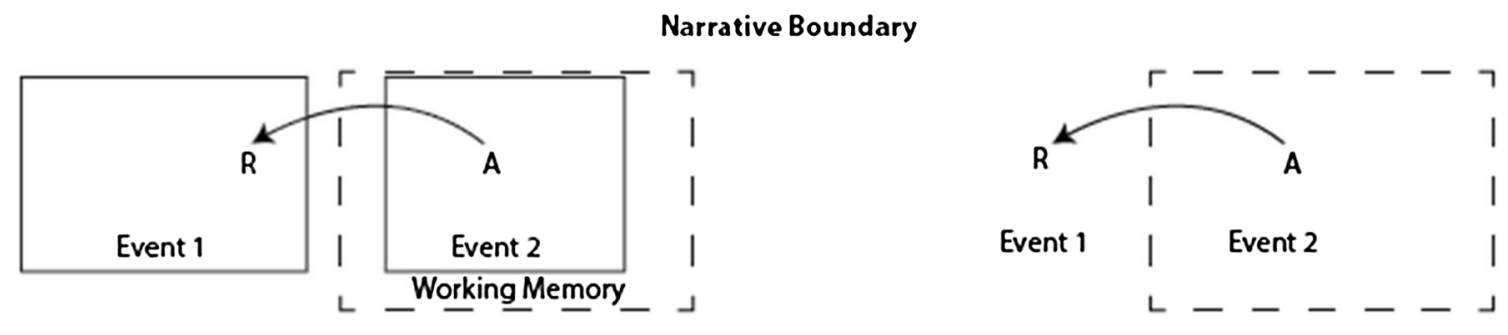

Distractor Task
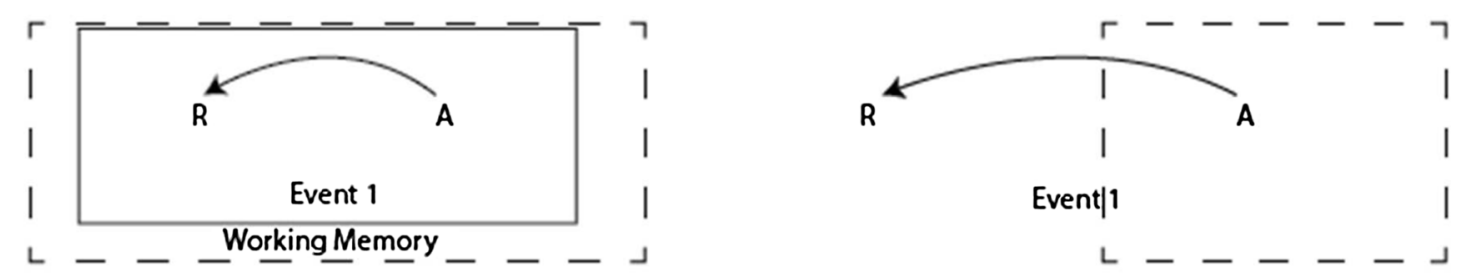

\section{Narrative Boundary and Distractor Task}
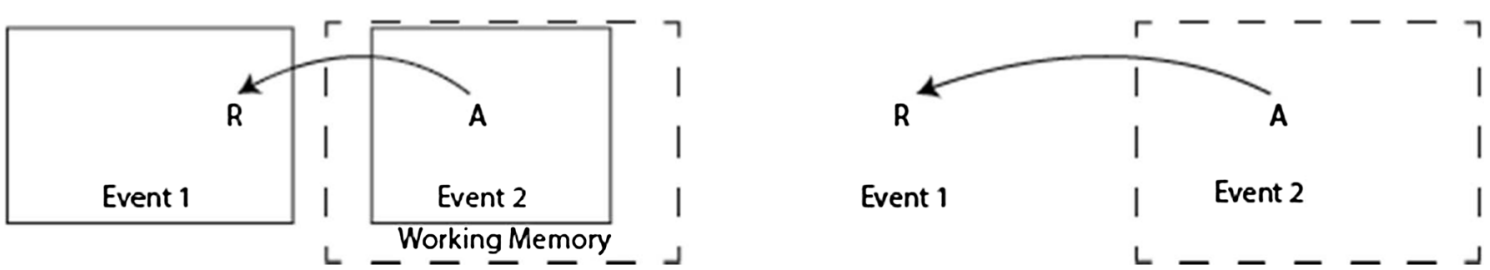

Fig. 1 Two possibilities for how event segmentation and working memory disruption can influence the availability of referent information. $\mathrm{R}=$ referent and $\mathrm{A}=$ anaphor

individual statements in the prior texts. When comprehension questions require a person to integrate across sentences that are on separate sides of a distractor task, then performance is worse (Foroughi, Werner, Barragán, \& Boehm-Davis, 2015). This last finding raises the possibility that readers may have difficulty when a distractor task separates the anaphor and referent, even if they are part of the same event.

To discriminate between these possibilities, we completed two experiments. Experiment 1 was a conceptual replication of the finding that narrative event boundaries impede the resolution of anaphors during reading. In Experiment 2, we replicated Experiment 1 but added the further manipulation of including a distractor task between the anaphor and the referent that served to largely clear working memory of thought related to the reading task. If anaphor disruption is due to just the narrative event boundary, then performance should be similar when reading in Experiment 2 involves a distractor task as when it does not. Alternatively, if anaphor disruption is due to a more general removal of information from working memory, and is not critically dependent on the presence of narrative event boundaries, then the presence of a distractor task should have a similar influence. 


\section{Experiment 1}

Experiment 1 assessed narrative event boundaries' influence on anaphoric reference, as has been reported previously (Anderson et al., 1983; Claus \& Kelter, 2006; Glenberg et al., 1987; Kelter et al., 2004; Radvansky \& Copeland, 2001). Participants read stories that contained an anaphor, in the form of repeated noun, which was preceded by the presence or absence of an event boundary between an anaphor and its referent.

\section{Method}

Participants Data were collected from 40 participants recruited from Amazon's Mechanical Turk service. Given the online nature of the study, we wanted to keep the number of materials and the length of participation relatively short. To adjust for the smaller number of stories, and thus the fewer number of observations per person, we increased the number of participants relative to most previously published work on this topic. Participants were paid $\$ 2.00$ for their participation, and the study was presented via the Qualtrics Survey Software website. Participants were limited to those who were over 18 and who were native English speakers located in the United States.

Materials Sixteen stories were used, four of which were filler stories and 12 of which were experimental stories. One of the experimental stories is shown in Table 1. The stories were 9 to 12 sentences in length. For each experimental narrative there was a referent sentence that contained the entity that would be referred to later. This was always followed by a filler sentence. Importantly, each experimental story had two versions that

Table 1 Sample story. The first sentence in bold contains the anaphor referent (dress, in this case). The second sentence in bold contains the event boundary and nonboundary versions of this sentence. Finally, the third sentence in bold contains the anaphor to the previously mentioned referent (dress, in this case)

The Hair Salon

The hairdresser, Marvin, picked up his scissors.

He started to cut small sections of hair.

The salon was filled with clients.

Rachel wanted a new hairstyle to go with the new dress she was wearing. (Referent sentence)

The hair salon was always busy during the day on weekends.

Rachel then had to go down to the nail salon./ Rachel thought about going to the nail salon. (Boundary sentence)

She did not end up liking her new hairstyle. (Buffer sentence)

The dress looked pretty though. (Anaphoric sentence)

Her date was supposed to pick her up at 5:00.

A friend had set them up.

Comprehension Question:

Rachel was at the hair salon.

True differed only in terms of one sentence. One sentence version conveyed an event boundary and the other did not. This is referred to as the boundary sentence. After the boundary sentence there was a buffer sentence, and then an anaphoric sentence containing the reference to that entity mentioned in the referent sentence (see Table 1).

In addition, there was a comprehension question at the end of each story that assessed various aspects of the stories and were answered by a true or false click response. These questions served as a gauge to make sure that the participants were actively reading the stories.

Procedure Participants were given a brief explanation of the study and a consent screen to approve on the Qualtrics website. Previous work has shown that web-based applications using JavaScript code, as we have done here, provides response-time estimates that are sufficiently accurate for the speed of processing being assessed here (Crump, McDonnell, \& Gureckis, 2013). After this, participants read instructions and continued on to the stories. At the beginning of each narrative, a story title was presented. Afterward, each story was presented one sentence at a time, and participants advanced through the story by pressing the space bar. Reading times were collected. After each story, the comprehension question was presented. Stories were presented in a random order for each person. Each participant saw an equal number of boundary and nonboundary stories. These were counterbalanced and randomized across participants.

Demographic questions were asked at the end of the experiment. This included gender, age, education level, ethnicity, handedness, environment, noise level, computing device, Internet connection, native language, and current state of alertness. Data from participants in noisy environments were excluded.

\section{Results and discussion}

Accuracy rates on the comprehension questions was high $(M=.94,95 \%$ CI $[.91, .96])$. The reading time data for the anaphor, referent, boundary, and buffer sentences were analyzed using a repeated measures ANOVA. Of most interest, for the anaphoric sentences, the effect of boundary condition was significant $F(1,39)=4.73, M S E=4,513, p=.04$, $\eta_{\mathrm{p}}{ }^{2}=.11$, with participants reading more slowly when an event boundary separated the anaphor and its referent (see Table 2). This replicates prior work (Anderson et al., 1983; Claus \& Kelter, 2006; Glenberg et al., 1987; Kelter et al., 2004; Radvansky \& Copeland, 2001). Thus, the narrative event boundaries served to impede memory, causing the anaphor resolution to take longer for the stories that included a narrative event boundary compared to those that did not. 
Table 2 Reading times (in ms/syllable) for the referent, boundary, and anaphor sentences in no-boundary and boundary conditions for Experiment 1. The $95 \%$ confidence intervals are reported in brackets

\begin{tabular}{lllll}
\hline Condition & Referent & Boundary & Buffer & Anaphor \\
\hline No boundary & $139[116,162]$ & $137[116,158]$ & $124[105,143]$ & $130[108,152]$ \\
Boundary & $148[122,174]$ & $153[118,187]$ & $127[109,145]$ & $158[122,194]$ \\
\hline
\end{tabular}

To assess whether performance on the anaphoric sentences could be due to other differences, such as the original encoding of the referent, the processing of the event boundary, or the buffer sentence, we also analyzed reading times for those differences. For the referent sentences, not surprisingly, the effect of boundary condition was not significant $F(1,39)=$ $0.49, M S E=3,468, p=.49, \eta_{\mathrm{p}}^{2}=.01$. Thus, the anaphoric sentence processing time effect is not due to differences in the original encoding of the referent.

For the boundary sentences, although reading times were nominally longer when there was an event boundary, this effect was not significant, $F(1,39)=1.36, M S E=3,523, p=.25$, $\eta_{\mathrm{p}}{ }^{2}=.03$. While many studies have found that event boundary sentences show an increase in reading time at those points (e.g., Zwaan et al., 1998), this is not always observed (e.g., Zwaan et al., 1995b). It has been shown, using memory probes, that people update their event models even when reading-time data show no such increase at these points (Radvansky \& Copeland, 2010).

For the buffer sentences, the effect of boundary condition was also not significant, $F(1,39)=0.29, M S E=618, p=.60$, $\eta_{\mathrm{p}}{ }^{2}=.005$. Thus, there was no difference in the reading times prior to the reading of the anaphoric sentence.

In sum, the results of Experiment 1 replicated prior work showing that the presence of a narrative event boundary made the resolution of an anaphoric reference more difficult. Based on an analysis of reading times for other sentences in the text, this is not likely due to factors other than the event boundary itself.

\section{Experiment 2}

The aim of Experiment 2 was to assess whether the anaphor disruption observed in Experiment 1 was due to the narrative event boundary segregating the information into separate event models (as suggested by the Event Horizon Model) or by a more general cognitive process that involves the removal of newly irrelevant information from working memory. To this end, we replicated the two conditions used in Experiment 1, along with two additional conditions. In one, rather than having a narrative event boundary, the anaphor and referent were separated by a distractor task (solving two-digit multiplication problems for 30 seconds) to clear out the contents of working memory (cf. Oulasvirta \& Saariluoma, 2006). In the other condition, both the narrative event boundary and the distractor task were present. So, in essence, a $2 \times 2$ design was used in which the presence or absence of a narrative event boundary was crossed with the presence or absence of a distractor task.

As noted in the introduction, there are two competing hypotheses (outlined in Fig. 1) about what might be causing the anaphor resolution disruption when there is a narrative event boundary, as was observed in Experiment 1. The first possibility is that the distractor task will not cause an anaphor resolution disruption. This pattern of data would be consistent with the Event Horizon Model idea that the disruption is caused by a narrative event boundary alone. That is, the narrative event boundary causes a new event model to be created. Information associated with the prior event model (in this case, the referent) would be associated with a prior event model, which is no longer maintained in working memory. In comparison, if a distractor task is presented that disrupts reading, then, although this task would remove the prior event model from working memory while the multiplication problems are being solved, after the distractor task was completed, the prior event model of the situation described by the text would be reactivated in working memory and performance would be similar to if there was no such disruption.

The second possibility is that the distractor task would also cause a disruption in anaphor resolution as part of a more general mechanism of removing information from working memory. Thus, once text information is removed from working memory by the distractor, including knowledge of the referent, it would need to be reactivated when the anaphor is encountered, regardless of the event structure. That is, performance would not be guided by the reactivation of a prior event model but by other nonevent-based processes that operate on the activation of concepts in memory in a more passive way.

\section{Method}

Participants We collected data from 80 participants, recruited using the Amazon Mechanical Turk service, who were paid $\$ 2.00$ for their participation. The larger sample size was a response to the increased number of conditions. Participants were limited to people who were over 18 years of age and were native English speakers located in the United States.

Materials and procedure The stories and procedure from Experiment 1 were used, except that for half of the stories there was a reading disruption that occurred after the 
boundary sentence in the form of a 30-s, two-digit math multiplication task (e.g., $61 \times 92=$ ?). For the distractor task, a reader was given 20 multiplication problems on a single screen. Readers scrolled down as they progressed through the problems. The distractor screen disappeared at the end of $30 \mathrm{~s}$, and the next text sentence was immediately presented. This distractor task occurred just after the boundary sentence, prior to the buffer sentence. The distractor task was counterbalanced across stories and participants. Thus, each person read three stories in each of the four conditions.

\section{Results and discussion}

Comprehension question accuracy rates were high $(M=.92$, $95 \%$ CI $[.90, .94])$. People solved an average of 3.1 (95\% CI $[2.83,3.41])$ multiplication problems during each distractor period.

The reading time data were analyzed using a 2 (narrative boundary) $\times 2$ (distractor task) repeated measures ANOVA. For the anaphor sentence, like Experiment 1, there was a main effect of boundary, with resolution taking longer if there was a narrative event boundary between the anaphor and the referent, $F(1,79)=4.29, M S E=6,314, p=.04, \eta_{\mathrm{p}}{ }^{2}=.05$ (see Table 3). Importantly, neither the main effect of distractor task, $F(1,79)=1.50, M S E=6,058, p=.22, \eta_{\mathrm{p}}{ }^{2}=.02$, nor the interaction were significant, $F(1,79)=0.01, M S E=6,351, p$ $=.94, \eta_{\mathrm{p}}{ }^{2}<.001$. Thus, the reading time increase in the event boundary conditions appears to be due to the separation of the anaphor and referent into different event models and does not appear to be primarily due to a removal of information from working memory apart from event structure, as is consistent with the Event Horizon Model account (Table 3).

To assess performance on whether the anaphoric sentences could be due to other differences, we also analyzed reading times for the referent, boundary, and buffer sentences. For the referent sentences, neither the main effects of boundary condition nor distractor were significant, $F(1,79)=2.83$, $M S E=7,085, p=.10, \eta_{\mathrm{p}}{ }^{2}=.03$, and $F(1,79)=0.03$, $M S E=5,636, p=.87, \eta_{\mathrm{p}}{ }^{2}<.001$, respectively, nor was the interaction, $F(1,79)=1.16, M S E=5,424, p=.29, \eta_{\mathrm{p}}{ }^{2}=.01$.
Thus, there were no major differences in the original encoding of the referent.

For the boundary sentences, neither the effect of boundary condition nor the distractor were significant, $F(1,79)=0.27$, $M S E=3,927, p=.60, \eta_{\mathrm{p}}{ }^{2}=.003$, and $F(1,79)=2.59, M S E=$ $3,361, p=.11, \eta_{\mathrm{p}}{ }^{2}=.03$, respectively, nor was the interaction, $F(1,79)=0.003, M S E=4,315, p=.96, \eta_{\mathrm{p}}{ }^{2}<.001$. Thus, like Experiment 1, no difference was observed in the reading times when the text conveyed an event boundary.

Finally, for the buffer sentences, while neither the main effect of boundary condition nor the interaction were significant, $F(1,79)=0.58, M S E=17,316, p=.45, \eta_{\mathrm{p}}{ }^{2}=.007$, and $F(1,79)=0.001, M S E=18,915, p=.98, \eta_{\mathrm{p}}{ }^{2}<.001$, respectively, there was a significant main effect of distractor, $F(1,79)$ $=104.34, M S E=68,729, p<.001, \eta_{\mathrm{p}}{ }^{2}=.60$. Specifically, unlike the subsequent anaphoric sentence, reading time was substantially slower if a participant had just completed the distractor task and was returning to the narrative. This is consistent with prior work showing a reading time increase when participants returned to a continuous text after an interruption as they reestablished their processing of the prior text (e.g., Fischer \& Glanzer, 1986; Glanzer et al., 1984).

In sum, the reading time data in Experiment 2 are most consistent with the idea that the disruption in anaphor processing following a narrative event boundary is due to the information being placed in a separate event model and not to a general loss of information from working memory. However, while the main effect of distractor presence was not significant, the reading times are in the general direction of greater difficulty of anaphor processing after a delay. It seems likely that under more extreme circumstances, such as a longer delay, some influence would be observed. However, nothing in this pattern of data indicates that this removal of the contents of working memory is contributing to the impairment of anaphor resolution caused by the narrative event boundary.

\section{General discussion}

The current experiments assessed whether the impairment of anaphor resolution in the presence of event boundaries during

Table 3 Reading times (in ms/syllable) for sentences containing anaphoric references in no-boundary and boundary conditions for Experiment 2. The $95 \%$ confidence intervals are reported in brackets.

\begin{tabular}{|c|c|c|c|c|}
\hline Condition & Referent & Boundary & Buffer & Anaphor \\
\hline \multicolumn{5}{|l|}{ Distractor absent } \\
\hline No boundary & $176[150,202]$ & $155[140,169]$ & $139[125,153]$ & $155[137,173$ \\
\hline Boundary & $151[138,165]$ & $151[134,168]$ & $150[127,172]$ & $173[152,194$ \\
\hline \multicolumn{5}{|l|}{ Distractor present } \\
\hline No boundary & $166[147,185]$ & $165[142,188]$ & $438[368,508]$ & $165[148,183$ \\
\hline Boundary & $159[136,182]$ & $161[140,182]$ & $450[383,517]$ & $184[153,216$ \\
\hline
\end{tabular}


narrative comprehension is due to the presence of narrative event boundaries or a more general removal of information from working memory. In Experiment 1, conceptually replicating prior work (Anderson et al., 1983; Claus \& Kelter, 2006; Glenberg et al., 1987; Kelter et al., 2004; Radvansky \& Copeland, 2001), we found that anaphor resolution was impaired, showing that the presence of a narrative event boundary did influence the accessibility of prior text information. Moreover, Experiment 2 assessed anaphor resolution in the presence or absence of both the narrative event boundary as well as a distractor task to clear working memory. The results showed that while the narrative event boundary had a significant influence on anaphor resolution reading times, the distractor task did not.

Overall, the results support the idea that when a narrative event boundary is encountered, readers engage in processes identified by Radvansky and Zacks (2014) as part of the Event Horizon Model. First, when a narrative event boundary is encountered, a new event model is created. Second, only the current event model is maintained in working memory. The prior event model, as well as the information associated with it, is removed from working memory. If reading is interrupted by some nonnarrative task, then the prior event model will be reactivated. In the case where there was no narrative event boundary, this will be the one event model that the person was working with previously, and the anaphor referent would be part of this event model in working memory. Alternatively, in the case where there was a narrative event boundary, the previous event model had already been removed from working memory, so the additional distractor task would have a relatively minor, if any, effect on later anaphor processing. This conclusion is supported by the finding that anaphor resolution was compromised by the presence of a narrative event boundary but not by the presence of a distractor task.

Having said this, two clarifications are needed. First, while in the current experiments the absence of a narrative event boundary resulted in a relatively easy resolution of an anaphor, this does not mean that anaphor resolution will be similar in all cases so long as the description is of one, singular event. This is clearly not the case. Instead, as descriptions of a single event grow more complex, it would be expected that this would also make the process of resolving an anaphor more difficult (e.g., Light \& Capps, 1986). Moreover, other, more linguistic factors could also have an influence, such as when there are multiple referents that could potentially be suitable for an anaphor (e.g., Gernsbacher \& Hargreaves, 1988).

Second, while in the current experiments the distractor task did not have an influence on anaphor resolution, this does not mean that longer or more involved interruptions would not. When a person turns away from the process of reading a text and then returns to it later, if the interruption is relatively mild and short-lived, then it would not be surprising that the person would be able to retrieve a prior event model into working memory, and it would be more or less intact. However, with longer intervals between the cessation of reading and picking it up again, one would expect the memory retrieval process to become more difficult and the integrity of the event models to be reduced with the passage of time. Thus, there are clear limits on the effects reported here.

Overall, and more generally, this work is consistent with the idea that as we progress through information in the world, in this case using written narrative, we parse our experience into events and remove information not relevant to current events, and this parsing influences how we perform. In many cases, this process may be advantageous in that when moving to a new event, the removal of previously relevant information may adjust cognition so that it is more suited to the current circumstances and is less distracted by elements that would have been relevant to the prior event. Problems arise when information remains relevant across events. These problems are not insurmountable, but disruptions can occur. This is also in line with the idea that events are a fundamental unit of cognition that helps structure and organize our thought.

\section{References}

Anderson, A., Garrod, S. C., \& Sanford, A. J. (1983). The accessibility of pronominal antecedents as a function of episode shifts in narrative text. Quarterly Journal of Experimental Psychology, 35, 427-440.

Bower, G. H., \& Morrow, D. G. (1990). Mental models in narrative comprehension. Science, 247, $44-48$.

Claus, B., \& Kelter, S. (2006). Comprehending narratives containing flashbacks: Evidence for temporally organized representations. Journal of Experimental Psychology: Learning, Memory, and Cognition, 32, 1031-1044.

Copeland, D. E., Magliano, J. P., \& Radvansky, G. A. (2006). Situation models in comprehension, memory, and augmented cognition. In C. Forsythe, M. L. Bernard, \& T. E. Goldsmith (Eds.), Human cognitive models in system design (pp. 37-66). Mahwah: Erlbaum.

Crump, M. J., McDonnell, J. V., \& Gureckis, T. M. (2013). Evaluating Amazon's Mechanical Turk as a tool for experimental behavioral research. PLoS ONE, 8(3), e57410.

Ezzyat, Y., \& Davachi, L. (2011). What constitutes an episode in episodic memory? Psychological Science, 22(2), 243-252.

Fischer, B., \& Glanzer, M. (1986). Short-term storage and the processing of cohesion during reading. The Quarterly Journal of Experimental Psychology, 38(3), 431-460.

Foroughi, C. K., Werner, N. E., Barragán, D., \& Boehm-Davis, D. A. (2015). Interruptions disrupt reading comprehension. Journal of Experimental Psychology: General, 144(3), 704-709.

Gernsbacher, M. A., \& Hargreaves, D. J. (1988). Accessing sentence participants: The advantage of first mention. Journal of Memory and Language, 27, 699-717.

Glanzer, M., Dorfman, D., \& Kaplan, B. (1981). Short-term storage in the processing of text. Journal of Verbal Learning and Verbal Behavior, 20(6), 656-670.

Glanzer, M., Fischer, B., \& Dorfman, D. (1984). Short-term storage in reading. Journal of Verbal Learning and Verbal Behavior, 23(4), 467-486. 
Glenberg, A. M., Meyer, M., \& Lindem, K. (1987). Mental models contribute to foregrounding during text comprehension. Journal of Memory and Language, 26, 69-83.

Kelter, S., Kaup, B., \& Claus, B. (2004). Representing a described sequence of events: A dynamic view of narrative comprehension. Journal of Experimental Psychology: Learning, Memory, and Cognition, 30, 451-464.

Kintsch, W. (1988). The role of knowledge in discourse comprehension: A construction-integration model. Psychological Review, 95(2), $163-182$

Lawrence, Z., \& Peterson, D. (2014). Mentally walking through doorways causes forgetting: The location updating effect and imagination. Memory (Ahead of print), 1-9.

Levine, W. H., \& Klin, C. M. (2001). Tracking of spatial information in narratives. Memory \& Cognition, 29, 327-335.

Light, L. L., \& Capps, J. L. (1986). Comprehension of pronouns in young and older adults. Developmental Psychology, 22, 580-585.

Magliano, J. P., \& Zacks, J. M. (2011). The impact of continuity editing in narrative film on event segmentation. Cognitive Science, 35(8), $1489-1517$

Magliano, J., Kopp, K., McNerney, M. W., Radvansky, G. A., \& Zacks, J. M. (2012). Aging and perceived event structure as a function of modality. Aging, Neuropsychology, and Cognition, 19(1/2), 264-282.

Morrow, D. G., Greenspan, S. L., \& Bower, G. H. (1987). Accessibility and situation models in narrative comprehension. Journal of Memory and Language, 26, 165-187.

Morrow, D. G., Bower, G., \& Greenspan, S. (1989). Updating situation models during narrative comprehension. Journal of Memory and Language, 28, 292-312.

Newtson, D. (1973). Attribution and the unit of perception of ongoing behavior. Journal of Personality and Social Psychology, 28(1), 28-38.

Oulasvirta, A., \& Saariluoma, P. (2006). Surviving task interruptions: Investigating the implications of long-term working memory theory. International Journal of Human-Computer Studies, 64(10), 941-961.

Radvansky, G. A. (2012). Across the event horizon. Current Directions in Psychological Science, 21, 4269-4272.

Radvansky, G. A., \& Copeland, D. E. (2001). Working memory and situation model updating. Memory \& Cognition, 29, 1073-1080.

Radvansky, G. A., \& Copeland, D. E. (2006). Walking through doorways causes forgetting: Situation models and experienced space. Memory \& Cognition, 35, 1150-1156.

Radvansky, G. A., \& Copeland, D. E. (2010). Reading times and the detection of event shift processing. Journal of Experimental Psychology. Learning, Memory, and Cognition, 36, 210-216.

Radvansky, G. A., \& Zacks, J. M. (2011). Event perception. Wiley Interdisciplinary Reviews: Cognitive Science, 2, 608-620.
Radvansky, G. A., \& Zacks, J. M. (2014). Event cognition. Boston: Oxford University Press.

Radvansky, G. A., Copeland, D. E., Berish, D. E., \& Dijkstra, K. (2003). Aging and situation model updating. Aging, Neuropsychology, and Cognition, 10(2), 158-166.

Radvansky, G. A., Tamplin, A. K., \& Krawietz, S. A. (2010). Walking through doorways causes forgetting: Environmental integration. Psychonomic Bulletin \& Review, 17, 900-904.

Radvansky, G. A., Krawietz, S. A., \& Tamplin, A. K. (2011). Walking through doorways causes forgetting: Further explorations. The Quarterly Journal of Experimental Psychology, 64, 1632-1645.

Radvansky, G. A., Pettijohn, K. A., \& Kim, J. (2015). Walking through doorways causes forgetting: Younger and older adults. Psychology and Aging, 30, 259-265.

Rinck, M., \& Bower, G. H. (1995). Anaphora resolution and the focus of attention in situation models. Journal of Memory and Language, 34, $110-131$.

Speer, N. K., \& Zacks, J. M. (2005). Temporal changes as event boundaries: Processing and memory consequences of narrative time shifts. Journal of Memory and Language, 53, 125-140.

Swallow, K. M., Zacks, J. M., \& Abrams, R. A. (2009). Event boundaries in perception affect memory encoding and updating. Journal of Experimental Psychology: General, 138, 236-257.

Tamplin, A. K., Krawietz, S. A., Radvansky, G. A., \& Copeland, D. E. (2013). Event memory and moving in a well-known environment. Memory \& Cognition, 41(8), 1109-1121.

Zacks, J., \& Tversky, B. (2001). Event structure in perception and cognition. Psychological Bulletin, 127, 3-21.

Zacks, J. M., Speer, N. K., \& Reynolds, J. R. (2009). Segmentation in reading and film comprehension. Journal of Experimental Psychology: General, 138(2), 307.

Zwaan, R. A. (1996). Processing narrative time shifts. Journal of Experimental Psychology: Learning, Memory, and Cognition, 22, 1196-1207.

Zwaan, R. A., \& Radvansky, G. A. (1998). Situation models in language comprehension and memory. Psychological Bulletin, 123(2), 162-185.

Zwaan, R. A., Langston, M. C., \& Graesser, A. C. (1995a). The construction of situation models in narrative comprehension: An eventindexing model. Psychological Science, 292-297.

Zwaan, R. A., Magliano, J. P., \& Graesser, A. C. (1995b). Dimensions of situation model construction in narrative comprehension. Journal of Experimental Psychology: Learning, Memory, and Cognition, 21(2), 386-397.

Zwaan, R. A., Radvansky, G. A., Hilliard, A. E., \& Curiel, J. M. (1998). Constructing multidimensional situation models during reading. Scientific Studies of Reading, 2(3), 199-220. 